\title{
INVARIANT DISTANCES RELATED TO THE BERGMAN FUNCTION
}

\author{
T. MAZUR, P. PFLUG AND M. SKWARCZYŃSKI
}

Abstract. Let $D$ be a bounded domain in $\mathbf{C}^{n}$. The invariant distance in $D$ is given by

$$
\rho_{D}(z, w)=\left(1-\left(\frac{K_{D}(z, w) K_{D}(w, z)}{K_{D}(z, z) K_{D}(w, w)}\right)^{1 / 2}\right)^{1 / 2}
$$

It is shown that one half of the length of a piecewise $C^{1}$ curve $\gamma:[a, b] \rightarrow D$ with respect to the Bergman metric is equal to the length of $\gamma$ measured by $\rho_{D}$, which implies that the associated inner distance $\rho_{D}^{*}$ coincides (up to the factor $\frac{1}{2}$ ) with the Bergman-distance. Also it was proved that $\rho_{D}$ is not an inner distance.

Introduction. Let $D$ be a bounded domain in $\mathbf{C}^{n}$. A distance function $\rho: D \times D \rightarrow$ $[0, \infty)$ can be used to define the length of a curve $\gamma:[a, b] \rightarrow D$ by the formula

$$
l_{\rho}(\gamma)=\sup \sum_{i=0}^{n-1} \rho\left(\gamma\left(t_{i}\right), \gamma\left(t_{i+1}\right)\right)
$$

where $a=t_{0}<t_{1} \cdots<t_{n}=b$ are arbitrary points on the segment $[a, b]$. For $p, q \in D$ let us define $\rho^{*}(p, q)$ as the greatest lower bound of the length of all curves in $D$ which join $p$ and $q$. Obviously $\rho^{*} \geqslant \rho$. The distance $\rho$ is called inner if $\rho^{*}=\rho$. The Bergman function $K_{D}(z, w), z, w \in D$ (see [1]), is related to the distance

$$
\rho_{D}(z, w)=\left(1-\left(\frac{K_{D}(z, w) K_{D}(w, z)}{K_{D}(z, z) K_{D}(w, w)}\right)^{1 / 2}\right)^{1 / 2}
$$

which induces the euclidean topology in $D$, and is invariant under biholomorphic mappings [6,7]. We shall also consider the Bergman distance $d_{D}(p, q), p, q \in D$, defined as the greatest lower bound of the Bergman length of all piecewise $C^{1}$ curves in $D$ which join $p$ and $q$. The Bergman length $l_{B}(\gamma)$ of $\gamma:[a, b] \rightarrow D$ is defined using the Bergman metric tensor

$$
d s^{2}=\sum_{j, k=1}^{N}\left(\frac{\partial^{2} \log K_{D}(z, z)}{\partial z_{j} \partial \bar{z}_{k}} d z_{j} \otimes d \bar{z}_{k}+\frac{\partial^{2} \log K_{D}(z, z)}{\partial \bar{z}_{j} \partial z_{k}} d \bar{z}_{j} \otimes d z_{k}\right) .
$$

Received by the editors January 20, 1984.

1980 Mathematics Subject Classification. Primary 32H15. 
Namely $\gamma^{*} d s^{2}=g(t) d t \otimes d t$ with $g(t)>0$, and

$$
\begin{aligned}
l_{B}(\gamma) & =\int_{a}^{b} g(t)^{1 / 2} d t \\
& =\sqrt{2} \int_{a}^{b}\left[\sum_{j, k} \frac{\partial^{2} \log K_{D}(\gamma(t), \gamma(t))}{\partial \bar{z}_{j} \partial z_{k}} \overline{\dot{\gamma}_{j}(t)} \dot{\gamma}_{k}(t)\right]^{1 / 2} d t
\end{aligned}
$$

The completeness of $d_{D}$ was investigated in $[2,3,4]$. In the present paper we shall prove the following results:

THEOREM 1. If $\gamma$ is a piecewise $C^{1}$ curve in $D$, then $l_{\rho_{D}}(\gamma)=\frac{1}{2} l_{B}(\gamma)$.

THEOREM 2. $\rho_{D}^{*}(p, q)=\frac{1}{2} d_{D}(p, q)$ for every $p, q \in D$.

This shows that the inner distance $\rho_{D}^{*}$ induced by $\rho_{D}$ is the integrated form of the Bergman-differential-metric (up to the factor $\frac{1}{2}$ ).

THEOREM 3. $\rho_{D}(p, q)<\rho_{D}^{*}(p, q)$ for every $p, q \in D, p \neq q$.

This implies $\rho_{D} \leqslant \frac{1}{2} d_{D}$; hence, $\rho_{D}$ completeness implies $d_{D}$ completeness, which was also shown in $[2,3]$. (The question whether $d_{D}$ completeness implies $\rho_{D}$ completeness is to our knowledge still open.) The third theorem says in particular that $\rho_{D}$ is not an inner distance.

1. Proof of Theorem 1. Using the general remarks in [5] it is enough to prove that if $\gamma:[0,1] \rightarrow D$ is a $C^{1}$-curve, then:

(A1) for $0 \leqslant s<1$ one has

$$
\lim _{t \searrow s} \frac{\rho_{D}(\gamma(t), \gamma(s))}{t-s}=\left[\frac{1}{2} \sum_{\nu, \mu=1}^{n} \frac{\partial^{2} \log K_{D}}{\partial z_{\nu} \partial \bar{z}_{\mu}}(\gamma(s), \gamma(s)) \cdot \dot{\gamma}_{\nu}(s) \cdot \overline{\dot{\gamma}_{\mu}(s)}\right]^{1 / 2}
$$

(Remark: the right side is continuous on $[0,1]$ ) and

(A2) for any $\varepsilon>0$ there exists $\delta>0$ such that if $0 \leqslant s<t \leqslant 1$ and $|t-s|<\delta$ one gets

$$
\left|\frac{\rho_{D}(\gamma(t), \gamma(s))}{t-s}-\left[\frac{1}{2} \sum_{\nu, \mu=1}^{n} \frac{\partial^{2} \log K_{D}}{\partial z_{\nu} \partial \bar{z}_{\mu}}(\gamma(s), \gamma(s)) \dot{\gamma}_{\nu}(s) \overline{\dot{\gamma}_{\mu}(s)}\right]^{1 / 2}\right|<\varepsilon .
$$

This can be shown with the use of Taylor's formula for the $C^{\infty}$-numerator $\phi(z, w)$ of

$$
\rho_{D}^{2}(z, w)=\frac{K_{D}(z, z) \cdot K_{D}(w, w)-K_{D}(z, w) \cdot K_{D}(w, z)}{\sqrt{K_{D}(z, z)} \cdot \sqrt{K_{D}(w, w)} \cdot\left[\sqrt{K_{D}(z, z)} \cdot \sqrt{K_{D}(w, w)}+\left|K_{D}(z, w)\right|\right]}
$$

taking into account that $\phi(z, w) \geqslant 0$ and $\phi(z, z)=0$. Hence this gives the proof of Theorem 1. 
2. Proof of Theorem $2{ }^{1}$ Denote by $\Gamma$ the family of all curves in $D$ which join $p$ and $q$. Denote by $\Gamma_{0}$ the subfamily of $\Gamma$ consisting of all piecewise $C^{1}$ curves. Then by Theorem 1

$$
\rho_{D}^{*}(p, q)=\inf _{\gamma \in \Gamma} l_{\rho_{D}}(\gamma) \leqslant \inf _{\gamma \in \Gamma_{0}} l_{\rho_{D}}(\gamma)=\frac{1}{2} d_{D}(p, q) .
$$

Assume $\rho_{D}^{*}(p, q)<A<A+\varepsilon_{0}<\frac{1}{2} d_{D}(p, q)$. Thus there exists a curve $\gamma_{0} \in \Gamma$ with

$$
l_{\rho_{D}}\left(\gamma_{0}\right)<A<A+\varepsilon_{0}<\inf _{\gamma \in \Gamma_{0}} l_{\rho_{D}}(\gamma) \text {. }
$$

Using the strong plurisubharmonicity of $\log K_{D}$ one immediately concludes that $\gamma_{0}$ is a rectifiable curve in the euclidean sense.

Analogous calculations as in (A1) and (A2) yield the following stronger result (compare Hilfssatz 2 in [5]): for any sequences $z^{\nu} \in D, w^{\nu} \in D, z^{\nu} \neq w^{\nu}$ with $\lim _{\nu \rightarrow \infty} z^{\nu}=\lim _{\nu \rightarrow \infty} w^{\nu}=z^{0} \in D$ and $\lim _{\nu \rightarrow \infty}\left(z^{\nu}-w^{\nu}\right) /\|z-w\|=v$ one has

$$
\frac{\rho_{D}\left(z^{\nu}, w^{\nu}\right)}{\left\|z^{\nu}-w^{\nu}\right\|} \rightarrow \alpha_{D}\left(z^{0} ; v\right):=\left[\frac{1}{2} \sum_{\nu, \mu=1}^{n} \frac{\partial^{2} \log K_{D}}{\partial z_{\nu} \partial \bar{z}_{\mu}}\left(z^{0}, z^{0}\right) v_{\nu} \bar{v}_{\mu}\right]^{1 / 2} .
$$

Then, for any $z^{0} \in D$ and any $\varepsilon>0$, there exists $\delta>0$ with the following property: whenever $z, w, \zeta \in D, z \neq w,\left\|z-z^{0}\right\|<\delta,\left\|w-z^{0}\right\|<\delta$ and $\left\|\zeta-z^{0}\right\|<\delta$, then

$$
\left|\frac{\rho_{D}(z, w)}{\|z-w\|}-\alpha_{D}\left(\zeta ; \frac{z-w}{\|z-w\|}\right)\right|<\varepsilon .
$$

Choose $\varepsilon:=\varepsilon_{0} / 2 L$ with $L$ equal to the euclidean length of $\gamma_{0}$. Using $(*)_{\varepsilon}$ one finds $\delta>0$ such that for any $t \in[0,1]$ the following property is true: for any $z \neq w, z, w$, $\zeta \in U\left(\gamma_{0}(t) ; \delta\right)=$ ball around $\gamma_{0}(t)$ with radius $\delta \subset D$ one has $(*)_{\varepsilon}$. This yields a partition $0=t_{0}<t_{1}<\cdots<t_{1}=1$ with

$$
\gamma_{0}\left(t_{i+1}\right) \in U\left(\gamma_{0}\left(t_{i}\right) ; \delta\right) \text {. }
$$

Setting $\beta_{i}:[0,1] \rightarrow U\left(\gamma_{0}\left(t_{i}\right) ; \delta\right)$ as the straight line from $\gamma_{0}\left(t_{i}\right)$ to $\gamma_{0}\left(t_{i+1}\right)$, Theorem 1 gives

$$
l_{\rho_{D}}\left(\beta_{i}\right)=\int_{0}^{1} \alpha_{D}\left(\beta_{i}(\tau) ; \gamma_{0}\left(t_{i+1}\right)-\gamma_{0}\left(t_{i}\right)\right) d \tau=\alpha_{D}\left(\beta_{i}\left(\tau_{i}\right) ; \gamma_{0}\left(t_{i+1}\right)-\gamma_{0}\left(t_{i}\right)\right)
$$

with $0 \leqslant \tau_{i} \leqslant 1$.

Combining the above remarks one receives

$$
\begin{aligned}
A \geqslant l_{\rho_{D}}\left(\gamma_{0}\right) \geqslant & \sum_{i=0}^{l-1} \rho_{D}\left(\gamma_{0}\left(t_{i}\right), \gamma_{0}\left(t_{i+1}\right)\right) \\
& \geqslant \sum_{i=0}^{l-1} \alpha_{D}\left(\beta_{i}\left(\tau_{i}\right), \gamma_{0}\left(t_{i}\right)-\gamma_{0}\left(t_{i+1}\right)\right)-\varepsilon \sum_{i=0}^{l-1}\left\|\gamma_{0}\left(t_{i}\right)-\gamma_{0}\left(t_{i+1}\right)\right\| \\
& \geqslant l_{\rho_{D}}(\beta)-\frac{\varepsilon_{0}}{2 L} \cdot L,
\end{aligned}
$$

\footnotetext{
${ }^{1}$ We thank Professor Reiffen for pointing out the importance of his Hilfssatz 2 of [5].
} 
where $\beta:[0,1] \rightarrow D$ is the polygon consisting of the $\beta_{i}$. Since $\beta \in \Gamma_{0}$ this gives a contradiction; hence, the proof of Theorem 2 is complete.

3. Proof of Theorem 3. First we shall prove the following general result.

Lemma 1. Consider $p, q \in D, p \neq q$. A distance $\rho$ in $D$ such that for all $w \in D$ the conditions, $w \neq p, w \neq q$ imply $\rho(p, q)<\rho(p, w)+\rho(w, q)$ and such that the function $w \rightarrow \rho(p, w)$ is continuous satisfies the inequality $\rho(p, q)<\rho^{*}(p, q)$.

Proof. Choose $r$ so small that the closed euclidean ball $\bar{U}(p ; r)$ satisfies the conditions

$$
q \notin \bar{U}(p, r), \quad \bar{U}(p, r) \subset D .
$$

Since the euclidean sphere is compact, there exists a positive $\varepsilon$ such that for all $w$ satisfying $\|w-p\|=r$

$$
(\rho(p, w)+\rho(w, q))-\rho(p, q) \geqslant \varepsilon .
$$

Every curve $\gamma \in \Gamma$ joins $p$ and $q$, and therefore intersects the boundary of $\bar{U}(p, r)$ at some point $w=\gamma\left(t_{1}\right), t_{1} \in[a, b]$. It follows that for every $\gamma \in \Gamma$

$$
l_{\rho}(\gamma) \geqslant \rho\left(p, \gamma\left(t_{1}\right)\right)+\rho\left(\gamma\left(t_{1}\right), q\right) \geqslant \rho(p, q)+\varepsilon .
$$

Therefore

$$
\rho^{*}(p, q)=\inf \left\{l_{\rho}(\gamma), \gamma \in \Gamma\right\} \geqslant \rho(p, q)+\varepsilon .
$$

The proof of the lemma is completed.

We shall now show that the assumptions of Lemma 1 hold for $\rho_{D}$ and every pair $p, q \in D, p \neq q$.

LemMA 2. For every $p, q, w \in D$ such that $p \neq q, w \neq p, w \neq q$

$$
\rho_{D}(p, q)<\rho_{D}(p, w)+\rho_{D}(w, q) .
$$

Proof. Denote by $\chi_{w}$ the unique element in the space $L^{2} H(D)$ (the Hilbert space of all square integrable holomorphic functions) which represents the evaluation functional $f \rightarrow f(w)$. By definition (see [6])

$$
\begin{aligned}
\rho_{D}(p, w) & =\frac{1}{\sqrt{2}} \inf _{\theta_{1}, \theta_{2} \in[0,2 \pi]}\left\|\frac{e^{i \theta_{1}} \cdot \chi_{p}}{\left\|\chi_{p}\right\|}-\frac{e^{i \theta_{2}} \cdot \chi_{w}}{\left\|\chi_{w}\right\|}\right\| \\
& =\frac{1}{\sqrt{2}} \inf _{\theta_{1} \in[0,2 \pi]}\left\|\frac{e^{i \theta_{1}} \cdot \chi_{p}}{\left\|\chi_{p}\right\|}-\frac{\chi_{w}}{\left\|\chi_{w}\right\|}\right\| .
\end{aligned}
$$

This infimum is attained at some point $\varphi \in[0,2 \pi]$. Hence

$$
\rho_{D}(p, w)=\frac{1}{\sqrt{2}}\left\|\frac{e^{i \varphi} \cdot \chi_{p}}{\left\|\chi_{p}\right\|}-\frac{\chi_{w}}{\left\|\chi_{w}\right\|}\right\|
$$

and similarily, there is $\Psi \in[0,2 \pi]$ such that

$$
\rho_{D}(q, w)=\frac{1}{\sqrt{2}}\left\|\frac{e^{i \Psi} \cdot \chi_{q}}{\left\|\chi_{q}\right\|}-\frac{\chi_{w}}{\left\|\chi_{w}\right\|}\right\| .
$$


Obviously

$$
\rho_{D}(p, q) \leqslant \frac{1}{\sqrt{2}}\left\|\frac{e^{i \rho} \cdot \chi_{p}}{\left\|\chi_{p}\right\|}-\frac{e^{i \Psi} \cdot \chi_{q}}{\left\|\chi_{q}\right\|}\right\| .
$$

Inequality (4) will be proved by contradiction. Assume that both sides in (4) are equal. This implies that the right side in (7) is the sum of right sides in (5) and (6). We know that if both sides in Minkowski inequality are equal, then the relevant vectors are linearly dependent. Therefore the functions $\chi_{p}, \chi_{q}, \chi_{w}$ (and the corresponding evaluation functionals) are linearly dependent. This is clearly impossible (evaluation functionals on the space of polynomials are linearly independent). This contradiction completes the proof of the lemma.

The proof of Theorem 3 follows directly from Lemmas 1 and 2.

\section{REFERENCES}

1. S. Bergman, The kernel function and conformal mapping, Math. Surveys, No. 5, Amer. Math. Soc., Providence, R. I., 1970.

2. S. Kobayashi, Geometry of bounded domains, Trans. Amer. Math. Soc. 92 (1959), 267-290.

3. P. Pflug, Various applications of the existence of well growing holomorphic functions, Functional Analysis, Holomorphy and Approximation Theory, Math. Studies, No. 71, North-Holland, Amsterdam, 1982, pp. 391-412.

4. T. Ohsawa, A remark on the completeness of the Bergman metric, Proc. Japan Acad. Ser. A Math. Sci. 57 (1981), 238-240.

5. H.-J. Reiffen, Die Carathéodorysche Distanz und ihre zugehörige Differentialmetrik, Math. Ann. 161 (1965), 315-324.

6. M. Skwarczynski, The invariant distance in the theory of pseudoconformal transformations and the Lu Qi-keng conjecture, Proc. Amer. Math. Soc. 22 (1969), 305-310.

7. B_ Biholomorphic invariants related to Bergman functions, Dissertationes Mathematici, no. 173, P.W.N., Warsaw, 1980.

Zakead Matematyki W. S. I., 26 - 600 Radom, Malczewskiego 29, Poland (Current address of M. Skwarczyński and T. Mazur)

Universität OSNabrUCK, Abteilung Vechta, 2848 Vechta, West Germany (Current address of P. Pflug) 